\title{
Image Segmentation by Image Foresting Transform with Boundary Polarity and Shape Constraints
}

\author{
Lucy A. C. Mansilla ${ }^{1}$ \\ Supervisor: Paulo A. V. Miranda ${ }^{1}$ \\ ${ }^{1}$ Department of Computer Science - University of São Paulo (USP) \\ 05508-090 - São Paulo - SP - Brazil \\ \{lucyacm, pmiranda\}@vision.ime.usp.br
}

\begin{abstract}
Image segmentation, such as to extract an object from a background, is very useful for medical and biological image analysis. In this work, we propose new segmentation methods for interactive segmentation of multidimensional images, based on the Image Foresting Transform (IFT), by exploiting for the first time non-smooth connectivity functions (NSCF) with a strong theoretical background. The new algorithms provide global optimum solutions according to an energy function of graph cut, subject to high-level boundary constraints (polarity and shape). Our experimental results indicate substantial improvements in accuracy in relation to other state-of-the-art methods, using medical images, by allowing the customization of the segmentation to a given target object.
\end{abstract}

\section{Introduction}

Image segmentation is one of the most fundamental and challenging problems in image processing and computer vision. In medical image analysis, accurate segmentation results commonly require the user intervention because of the presence of structures with ill-defined borders, intensity non-standardness among images, field inhomogeneity, noise, artifacts, partial volume effects, and their interplay. The high-level, application-domain-specific knowledge of the user is also often required in the digital matting of natural scenes, because of their heterogeneous nature. These problems motivated the development of several methods for semi-automatic segmentation, aiming to minimize the user involvement and time required without compromising accuracy and precision [Mansilla 2014].

One important class of interactive image segmentation comprises seed-based methods, which have been developed based on different theories, supposedly not related, leading to different frameworks, such as watershed, random walks, fuzzy connectedness, graph cuts, distance cut, image foresting transform (IFT) [Falcão et al. 2004], and grow cut. The study of the relations among different frameworks, including theoretical and empirical comparisons, has a vast literature. However, these methods in most studies are restricted to undirected graphs, and the most time-efficient methods, including the IFT, present a lack of boundary regularization constraints.

The main contribution of this work is a theoretical development to support the usage of non-smooth connectivity functions (NSCF) in the IFT, opening new perspectives in the research of image processing using graphs, since NSCF were, until now, avoided in the literature. More specifically, we prove that some NSCF can lead to optimum results according to a graph-cut measure on a digraph [Miranda and Mansilla 2014, 
Mansilla and Miranda 2013a]. This oriented energy allows the incorporation of the boundary polarity and shape constraints, to eliminate false delineations, as discussed later on Sections 3 and 4. We have as main results:

1. The customization of the segmentation by IFT to match the global features of a target object: (a) The orientation of the object's intensity transitions, i.e., bright to dark or the opposite (boundary polarity), and (b) shape constraints to regularize the segmentation boundary (geodesic star convexity constraint).

2. The development of an interactive segmentation tool within the software, called Brain Image Analyzer (BIA), to support research in neurology involving volumetric magnetic resonance images of a 3T scanner from the FAPESP-CInApCe.

3. Four conference papers were published [Mansilla and Miranda 2013a, Mansilla et al. 2013b, Mansilla and Miranda 2013b, Mansilla et al. 2013a], and one journal paper was published in the IEEE Transactions on Image Processing (impact factor: 3.111) [Miranda and Mansilla 2014].

\section{Image Foresting Transform (IFT)}

An image 2D/3D can be interpreted as a weighted digraph $G=\langle\mathcal{V}=\mathcal{I}, \boldsymbol{\xi}, \omega\rangle$ whose nodes $\mathcal{V}$ are the image pixels in its image domain $\mathcal{I} \subset \mathbb{Z}^{N}$, and whose arcs are the ordered pixel pairs $(s, t) \in \boldsymbol{\xi}$. The digraph $G$ is symmetric if for any of its arcs $(s, t)$, the pair $(t, s)$ is also an arc of $G$. We use $(s, t) \in \boldsymbol{\xi}$ to indicate that $t$ is adjacent to $s$. Each arc $(s, t) \in \boldsymbol{\xi}$ may have a weight $\omega(s, t) \geq 0$, such as a dissimilarity measure between pixels $s$ and $t$ (e.g., $\omega(s, t)=|I(t)-I(s)|$ for a single channel image with values given by $I(t)$ ). For a given image graph $G$, a path $\pi_{t}=\left\langle t_{1}, t_{2}, \ldots, t_{n}=t\right\rangle$ is a sequence of adjacent pixels with terminus at a pixel $t$. A path is trivial when $\pi_{t}=\langle t\rangle$. A path $\pi_{t}=\pi_{s} \cdot\langle s, t\rangle$ indicates the extension of a path $\pi_{s}$ by an $\operatorname{arc}(s, t)$. The notation $\pi_{s \backsim t}=\left\langle t_{1}=s, t_{2}, \ldots, t_{n}=t\right\rangle$ may also be used, where $s$ stands for the origin and $t$ for the destination node. A connectivity function computes a value $f\left(\pi_{t}\right)$ for any path $\pi_{t}$, usually based on arc weights. A path $\pi_{t}$ is optimum if $f\left(\pi_{t}\right) \leq f\left(\tau_{t}\right)$ for any other path $\tau_{t}$ in $G$. The IFT takes an image graph $G$, and a path-cost function $f$; and assigns one optimum path $\pi_{t}$ to every pixel $t \in \mathcal{V}$ such that an optimum-path forest $P$ is obtained, i.e., a spanning forest where all induced paths are optimum. However, $f$ must be smooth, otherwise, the paths may not be optimum [Falcão et al. 2004]. In the forest, each object is represented by the optimum-path trees rooted at its internal seeds.

\section{Boundary Polarity via NSCF}

In order to resolve between very similar nearby boundary segments, in [Miranda and Mansilla 2014, Mansilla and Miranda 2013a] we successfully incorporated the boundary polarity constraint in the IFT using NSCF in digraphs, resulting in a novel method called Oriented Image Foresting Transform (OIFT).

In the case of digraphs, there are two different types of cut for each object boundary: an inner-cut boundary composed by arcs that point toward object pixels $\mathcal{C}_{\boldsymbol{i}}(L)=\{(s, t) \in \boldsymbol{\xi} \mid L(s)=0, L(t)=1\}^{1}$, and an outer-cut boundary with arcs from object to background pixels $\mathcal{C}_{\boldsymbol{o}}(L)=\{(s, t) \in \boldsymbol{\xi} \mid L(s)=1, L(t)=0\}$. Consequently,

\footnotetext{
${ }^{1} L(t)=1$ and $L(t)=0$ represent object and background pixels in a labeled image $L$, respectively.
} 
we consider two different types of energy, $E_{i}$ (Eq. 1) and $E_{o}$ (Eq.2).

$$
\begin{aligned}
& E_{i}(L, G)=\min _{(s, t) \in \mathcal{C}_{i}(L)} \omega(s, t) \\
& E_{o}(L, G)=\min _{(s, t) \in \mathcal{C}_{o}(L)} \omega(s, t)
\end{aligned}
$$

We use a digraph, where $\omega(s, t)$ is a combination of a regular undirected dissimilarity measure $\psi(s, t)$, multiplied by an orientation factor (i.e., $\omega(s, t)=\psi(s, t) \times(1+\alpha)$ if $I(s)>I(t)$ and $\omega(s, t)=\psi(s, t) \times(1-\alpha)$ otherwise). Several different procedures can be adopted for $\psi(s, t)$, such as the absolute value of the difference of image intensities (i.e., $\psi(s, t)=|I(t)-I(s)|)$. Note that we have $\omega(s, t) \neq \omega(t, s)$ when $\alpha>0$.

The OIFT is build upon the IFT framework by considering one of the following path functions in a symmetric digraph:

$$
\begin{aligned}
& f_{\max }^{\mathcal{S}_{1}, \mathcal{S}_{2}}(\langle t\rangle)= \begin{cases}-1 & \text { if } t \in \mathcal{S}_{1} \cup \mathcal{S}_{2} \\
+\infty & \text { otherwise }\end{cases} \\
& f_{\max }^{\mathcal{S}_{1}, \mathcal{S}_{2}}\left(\pi_{r \rightsquigarrow s} \cdot\langle s, t\rangle\right)= \begin{cases}\max \left\{f_{\max }^{\mathcal{S}_{1}, \mathcal{S}_{2}}\left(\pi_{r \rightsquigarrow s}\right), 2 \times \omega(t, s)+1\right\} & \text { if } r \in \mathcal{S}_{1} \\
\max \left\{f_{\max }^{\mathcal{S}_{1}, \mathcal{S}_{2}}\left(\pi_{r \rightsquigarrow s}\right), 2 \times \omega(s, t)\right\} & \text { if } r \in \mathcal{S}_{\mathbf{2}}\end{cases} \\
& f_{\omega}^{\mathcal{S}_{1}, \mathcal{S}_{2}}(\langle t\rangle)=f_{\max }^{\mathcal{S}_{1}, \mathcal{S}_{2}}(\langle t\rangle) \\
& f_{\omega}^{\mathcal{S}_{1}, \mathcal{S}_{2}}\left(\pi_{r \rightsquigarrow s} \cdot\langle s, t\rangle\right)= \begin{cases}\omega(t, s) & \text { if } r \in \mathcal{S}_{1} \\
\omega(s, t) & \text { if } r \in \mathcal{S}_{\mathbf{2}}\end{cases}
\end{aligned}
$$

The segmentation using $f_{\max }^{\mathcal{S}_{o}, \mathcal{S}_{b}}$ or $f_{\omega}^{\mathcal{S}_{o}, \mathcal{S}_{b}}$ favors transitions from dark to bright pixels, and $f_{\max }^{\mathcal{S}_{b}, \mathcal{S}_{o}}$ or $f_{\omega}^{\mathcal{S}_{b}, \mathcal{S}_{o}}$ favors the opposite orientation, according to Theorem 1, where $\mathcal{S}_{o}$ is a set of object's seeds and $\mathcal{S}_{b}$ are seeds selected in the background. In the case of multiple candidate segmentations with the same energy, $f_{\omega}^{\mathcal{S}_{1}, \mathcal{S}_{2}}$ produces a better handling of the tie zones than $f_{\max }^{\mathcal{S}_{1}, \mathcal{S}_{2}}$ [Mansilla and Miranda 2013a].

Theorem 1 (Inner/outer-cut boundary optimality) For two given sets of seeds $\mathcal{S}_{o}$ and $\mathcal{S}_{b}$, any spanning forest computed by the IFT algorithm for function $f_{\max }^{\mathcal{S}_{b}, \mathcal{S}_{o}}$ or $f_{\omega}^{\mathcal{S}_{b}, \mathcal{S}_{o}}$ defines an optimum cut that maximizes $E_{o}(L, G)$ among all possible segmentation results satisfying the hard constraints. Any spanning forest computed by the IFT algorithm for function $f_{\max }^{\mathcal{S}_{o}, \mathcal{S}_{b}}$ or $f_{\omega}^{\mathcal{S}_{o}, \mathcal{S}_{b}}$ defines an optimum cut that maximizes $E_{i}(L, G)$ among all possible segmentation results satisfying the hard constraints.

In our experiments, we used 20 volumetric images from real 3D MR images of the foot. We computed the mean performance curve (Dice coefficient) for the methods: Iterative Relative Fuzzy Connectedness (IRFC), IFT with $f_{\max }$ [Falcão et al. 2004] $\left(I F T_{F I F O}^{\max }\right)$, Power Watershed $\left(P W_{q=2}\right)$, and OIFT using $f_{\max }^{\mathcal{S}_{o}, \mathcal{S}_{b}}\left(O I F T_{\text {inner }}^{\max }\right), f_{\max }^{\mathcal{S}_{b}, \mathcal{S}_{o}}$ $\left(O I F T_{\text {outer }}^{\max }\right), f_{\omega}^{\mathcal{S}_{o}, \mathcal{S}_{b}}\left(O I F T_{\text {inner }}^{\omega}\right)$ and $f_{\omega}^{\mathcal{S}_{b}, \mathcal{S}_{o}}\left(O I F T_{\text {outer }}^{\omega}\right)$ [Mansilla 2014]. We used different seed sets obtained by eroding and dilating the ground truth (Figure 1). The experimental accuracy curves with the Sobel gradient (Figure 2) show that whenever the object presents transitions from dark to bright pixels, as it is the case with the bones, $f_{\omega}^{\mathcal{S}_{o}, \mathcal{S}_{b}}$ and $f_{\max }^{\mathcal{S}_{o}, \mathcal{S}_{b}}$ give the best accuracy results. Note also that $f_{\max }^{\mathcal{S}_{b}, \mathcal{S}_{o}}$ and $f_{\omega}^{\mathcal{S}_{b}, \mathcal{S}_{o}}$ present the worst accuracy values, by specifying the wrong orientation.

\section{Shape Constraints via NSCF}

Shape constraints, such as the star-convexity prior introduced by Veksler [Veksler 2008], can limit the search space of possible delineations to a smaller subset, thus eliminating 


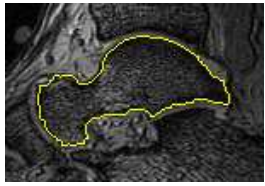

(a)

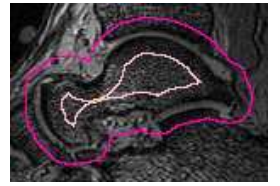

(b)

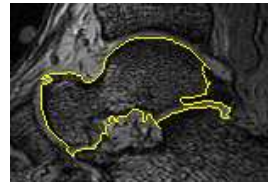

(c)

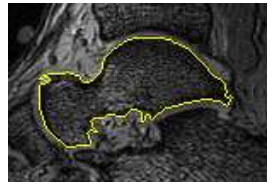

(d)

Figure 1. (a) True segmentation of the talus bone in an MR image of a foot. (b) Seed sets obtained by eroding and dilating the ground truth. (c) Segmentation by IRFC. (d) An improved result by exploiting the boundary polarity using $f_{\max }^{\mathcal{S}_{o}, \mathcal{S}_{b}}$.

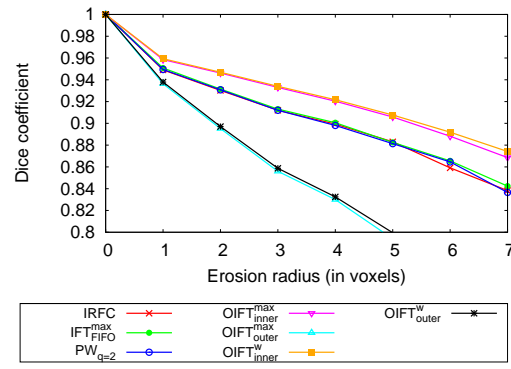

(a)

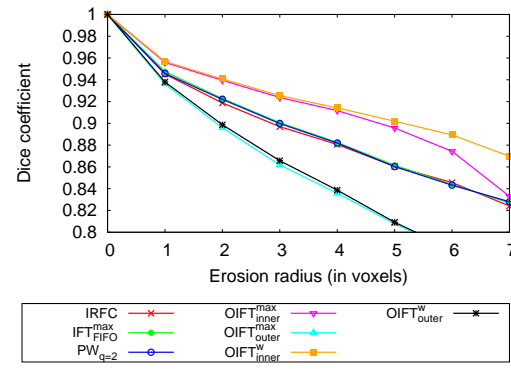

(b)

Figure 2. The mean accuracy curves (Dice) using the Sobel gradient for the 3D segmentation of: (a) talus, and (b) calcaneus.

false candidate boundaries. In this context, a point $p$ is said to be visible to $c$ via a set $\mathcal{O}$ if the line segment joining $p$ to $c$ lies in the set $\mathcal{O}$. An object $\mathcal{O}$ is star-convex with respect to center $c$, if every point $p \in \mathcal{O}$ is visible to $c$ via $\mathcal{O}$ (Figure 3 ). In the case of multiple stars, a computationally tractable definition, was proposed in [Gulshan et al. 2010], using a Geodesic Star Convexity (GSC) constraint in the segmentation by min-cut/max-flow.

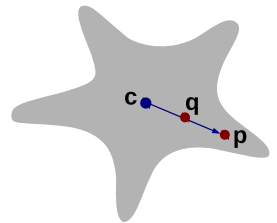

(a)

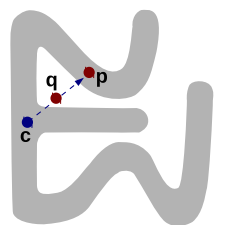

(b)

Figure 3. For any point $p$ within the object and the star center $c$, we have: (a) a star-convex object and (b) a non-star-convex object.

In [Mansilla et al. 2013b], we proposed an IFT extension that incorporates the GSC constraint, favoring the segmentation of objects with more regular shape, resulting in a novel method called IFT with Geodesic Star Convexity Constraints (GSC-IFT). In this method, the set of star centers is taken as the set of internal seeds $\left(\mathcal{S}_{o}\right)$, and the line segments are the paths that form a spanning forest rooted at the internal seeds. The forest topology is controlled by a parameter $\beta$. For lower values of $\beta(\beta \approx 0.0)$, the method imposes more star regularization to the boundary of the object, and for higher values of $\beta$, it allows a better fit to the curved protrusions and indentations of the boundary.

Thereafter, in [Mansilla and Miranda 2013b] we proposed the novel method called OIFT with Geodesic Star Convexity (GSC-OIFT), which incorporate Gulshan's 
geodesic star convexity prior in the OIFT approach for interactive image segmentation, in order to simultaneously handle boundary polarity and shape constraints (Theorem 2). We constrain the search for optimum result, that maximize the graph-cut measures $E_{i}$ (Eq. 1) or $E_{o}$ (Eq. 2), only to segmentations that satisfy the geodesic star convexity constraint. We compute an optimum forest $P_{\text {sum }}$ for $f_{\text {sum }}$ [Falcão et al. 2004] by the regular IFT algorithm, using only $\mathcal{S}_{o}$ as seeds, for the given digraph $G$, obtaining two sets of arcs $\boldsymbol{\xi}_{\boldsymbol{P}_{\text {sum }}}^{i}=\left\{(s, t) \in \boldsymbol{\xi} \mid s=P_{\text {sum }}(t)\right\}$ and $\boldsymbol{\xi}_{\boldsymbol{P}_{\text {sum }}}^{o}=\left\{(s, t) \in \boldsymbol{\xi} \mid t=P_{\text {sum }}(s)\right\}$.

Theorem 2 (Inner/outer-cut boundary optimality) For a given image graph $G=\langle\mathcal{I}, \boldsymbol{\xi}, \omega\rangle$, consider a modified weighted graph $G^{\prime}=\left\langle\mathcal{I}, \boldsymbol{\xi}, \omega^{\prime}\right\rangle$, with weights

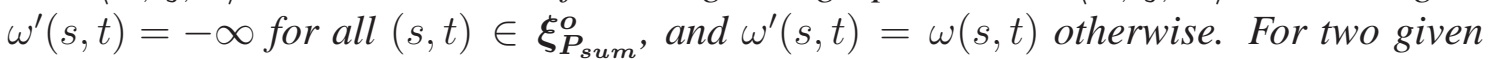
sets of seeds $\mathcal{S}_{o}$ and $\mathcal{S}_{b}$, the segmentation computed over $G^{\prime}$ by the IFT algorithm for function $f_{\max }^{\mathcal{S}_{b}, \mathcal{S}_{o}}$ defines an optimum cut in the original graph $G$, that maximizes $E_{o}(L, G)$ among all possible segmentation results satisfying the shape constraints by the geodesic star convexity, and the seed constraints. Similarly, the segmentation computed by the IFT algorithm for function $f_{\max }^{\mathcal{S}_{o}, \mathcal{S}_{b}}$, over a modified graph $G^{\prime}=\left\langle\mathcal{I}, \boldsymbol{\xi}, \omega^{\prime}\right\rangle$; with weights $\omega^{\prime}(s, t)=-\infty$ for all $(s, t) \in \boldsymbol{\xi}_{P_{\text {sum }}}^{i}$, and $\omega^{\prime}(s, t)=\omega(s, t)$ otherwise; defines an optimum cut in the original graph $G$, that maximizes $E_{i}(L, G)$ among all possible segmentation results satisfying the shape constraints by the geodesic star convexity.

In our experiments, we used 40 image slices of 10 thoracic CT studies to segment the liver. Figure 4a shows the mean accuracy curves for all the images assuming different seed sets obtained by eroding and dilating the ground truth. Note that for higher values of $\beta$, GSC-OIFT imposes less shape constraints, so that the accuracy tends to decrease (Figures $4 \mathrm{~b}-\mathrm{c}$ ). Figure 5 shows some results in the case of user-selected markers.

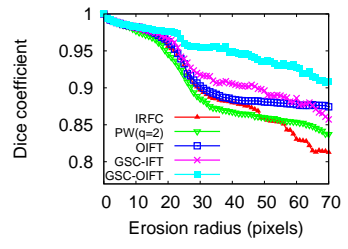

(a)

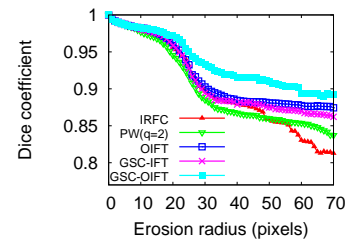

(b)

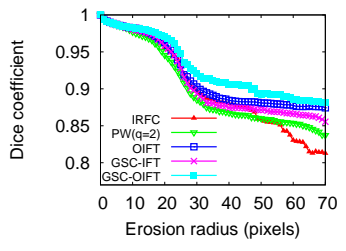

(c)

Figure 4. The mean accuracy curves of all methods for the liver segmentation for various values of $\beta$ : (a) $\beta=0.0$, (b) $\beta=0.5$, and (c) $\beta=0.7$.

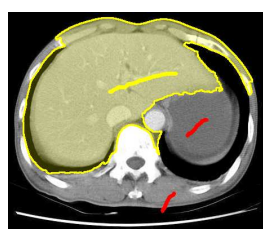

(a)

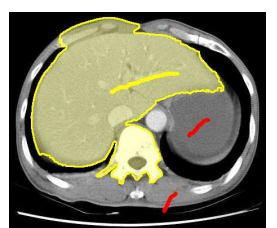

(b)

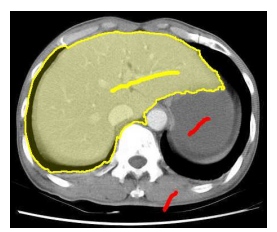

(c)

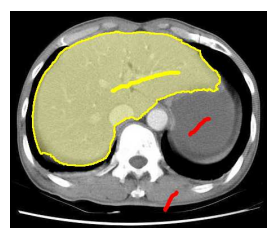

(d)

Figure 5. Results for user-selected markers: (a) IRFC, (b) OIFT ( $f_{\max }^{\mathcal{S}_{b}, \mathcal{S}_{o}}$ with $\alpha=0.5$ ), (c) GSC-IFT ( $\beta=0.7, \alpha=0.0$ ), and (d) GSC-OIFT ( $\beta=0.7, \alpha=0.5$ ).

\section{Conclusion}

The proposed extension GSC-OIFT includes the IFT with $f_{\max }$, OIFT and GSC-IFT as particular cases, depending on the configuration of its parameters $\alpha$ and $\beta$. Other result 
of our work was the design of more adaptive and flexible connectivity functions, with the use of dynamic weights, that allow better handling of images with strong inhomogeneity [Mansilla et al. 2013a]. The theoretical foundation proposed in this work has also allowed new achievements that were recently published, such as [Braz and Miranda 2014] and [Bejar and Miranda 2014].

The authors thank FAPESP grant \#2011/50761-2 \#2012/06911-2, CNPq, CAPES, NAP eScience - PRP - USP and Dr. J.K. Uduapa (MIPG-UPENN) for the images.

\section{References}

Bejar, H. and Miranda, P. (2014). Oriented relative fuzzy connectedness: Theory, algorithms, and applications in image segmentation. In XXVII Conference on Graphics, Patterns and Images (SIBGRAPI), pages 304-311, Rio de Janeiro, Brazil. IEEE.

Braz, C. and Miranda, P. (2014). Image segmentation by image foresting transform with geodesic band constraints. In IEEE International Conference on Image Processing (ICIP), pages 4333 - 4337, Paris, France.

Falcão, A., Stolfi, J., and Lotufo, R. (2004). The image foresting transform: Theory, algorithms, and applications. IEEE Transactions on Pattern Analysis and Machine Intelligence, 26(1):19-29.

Gulshan, V., Rother, C., Criminisi, A., Blake, A., and Zisserman, A. (2010). Geodesic star convexity for interactive image segmentation. In Proceedings of Computer Vision and Pattern Recognition, pages 3129-3136.

Mansilla, L. (2014). Image foresting transform with non-smooth connectivity functions: Adaptive weights, boundary polarity, and shape constraints. Master's thesis, Institute of Mathematics and Statistics, University of São Paulo, Brazil. http://www.teses.usp.br/teses/disponiveis/45/45134/tde-17032014-121734/pt-br.php.

Mansilla, L., Cappabianco, F., and Miranda, P. (2013a). Image segmentation by image foresting transform with non-smooth connectivity functions. In XXVI Conference on Graphics, Patterns and Images (SIBGRAPI), pages 147-154, Arequipa, Perú. IEEE.

Mansilla, L., Jackowski, M., and Miranda, P. (2013b). Image foresting transform with geodesic star convexity for interactive image segmentation. In IEEE International Conference on Image Processing (ICIP), pages 4054-4058, Melbourne, Australia.

Mansilla, L. and Miranda, P. (2013a). Image segmentation by oriented image foresting transform: Handling ties and colored images. In 18th International Conference on Digital Signal Processing (DSP), pages 1-6, Santorini, Greece. IEEE.

Mansilla, L. and Miranda, P. (2013b). Image segmentation by oriented image foresting transform with geodesic star convexity. In Computer Analysis of Images and Patterns $(C A I P)$, volume 8047, pages 572-579, York, UK.

Miranda, P. and Mansilla, L. (2014). Oriented image foresting transform segmentation by seed competition. IEEE Transactions on Image Processing, 23(1):389-398.

Veksler, O. (2008). Star shape prior for graph-cut image segmentation. In European Conference on Computer Vision (ECCV), volume 5304, pages 454-467. 